\title{
Operation of the DC Current Transformer intensity monitors at FNAL during Run II $^{1}$
}

\section{J. Crisp, ${ }^{a, 2}$ B. Fellenz, ${ }^{a}$ D. Heikkinen, ${ }^{a}$ M.A. Ibrahim, ${ }^{a, 3}$ T. Meyer ${ }^{a}$ and G. Vogel ${ }^{a}$}

Fermi National Accelerator Laboratory,

Batavia, IL, U.S.A.

E-mail: cadornaa@fnal.gov

ABSTRACT: Circulating beam intensity measurements at FNAL are provided by five DC current transformers (DCCT), one per machine. With the exception of the DCCT in the Recycler, all DCCT systems were designed and built at FNAL. This paper presents an overview of both DCCT systems, including the sensor, the electronics, and the front-end instrumentation software, as well as their performance during Run II.

KEYWORDS: Instrumentation for particle accelerators and storage rings - high energy (linear accelerators, synchrotrons); Beam-line instrumentation (beam position and profile monitors; beamintensity monitors; bunch length monitors)

\footnotetext{
${ }^{1}$ Work supported by Fermi Research Alliance, LLC under Contract No. DE-AC02-07CH11359 with the United States Department of Energy.

${ }^{2}$ Now at Michigan State University.

${ }^{3}$ Corresponding author.
} 


\section{Contents}

1 Introduction 1

2 DCCT operational theory 2

2.1 Magnetic modulator circuit 2

2.1.1 Pure ac excitation 2

2.1.2 Excitation with dc bias 2

2.2 Application of bucking current 6

2.3 Extending system bandwidth 6

3 DCCT systems installed at FNAL $\quad 7$

3.1 Custom-built detector 9

3.2 Commercial detector 10

4 Control system interface $\quad 12$

$\begin{array}{lll}\text { 4.1 VME environment } & 12\end{array}$

$\begin{array}{ll}4.2 \text { Continuous intensity measurements } & 12\end{array}$

4.3 Event-timed intensity measurements 12

$\begin{array}{lll}4.4 & \text { Beam loss-rate measurements } & 13\end{array}$

5 Operational performance $\quad 13$

5.1 RMS noise observation 13

$\begin{array}{ll}5.2 \text { Nonlinearity studies } & 15\end{array}$

5.3 Bunch length sensitivity 16

6 Conclusion 18

\section{Introduction}

An essential part of generating and controlling charged particle beams is a precise measurement of the intensity of the beam. There are a variety of ways of accomplishing this task using capacitively or inductively coupled signals as well as outright absorption of the beam [1]-[3]. The problem with most techniques is that they are narrow band, or at the very least incapable of measuring dc intensity. One device is a current transformer involving a magnetic modulator, which typically called a dc current transformer (DCCT) [4]-[7]. A DCCT intensity monitor provides non-destructive, accurate, and stable measurements of the beam intensity of circulating beams over a large dynamic range. 


\section{DCCT operational theory}

A DCCT is part of a subset of electromagnetic beam monitors that rely primarily on interaction with the magnetic field. The system consists of a pair of vacuum beam pipes separated by an insulating ceramic piece and highly permeable magnetic cores which, altogether, function as an ac transformer, complete with low frequency cut-off. However, a DCCT system extends its useful bandwidth past the normal lower cut-off frequency of the core, by utilizing a magnetic modulator and exploiting the hysteresis curve of the material.

\subsection{Magnetic modulator circuit}

An ac excitation signal will modulate the magnetic cores at a frequency and power which is dependent on the cores' non-linear characteristics. For a given magnetic material of predetermined physical dimensions, the core power loss is inversely related to the excitation frequency, and the power of the excitation signal must drive the cores deep into saturation to insure no remnant field accumulate. Driving the cores hard into saturation allows the feedback loop to sense the addition of even a relatively small dc bias.

The magnetic modulator can be seen as a magnetic mixer shifting the beam signal frequency spectrum by twice the modulation frequency. The modulation generator must have either high current or high voltage capabilities as well as maintain frequency spectrum purity with a highly non-linear load.

\subsubsection{Pure ac excitation}

When a single core is excited under pure ac conditions, its hysteresis curve is symmetrical with respect to the magnetic flux density (B) and magnetic field strength $(\mathrm{H})$ axes. As the cores are driven into saturation, the induced output in the pickup is clamped. Hence, the frequency spectrum of the induced output in the pickup winding presents only odd harmonics.

Moreover, counter-phased winding of two matched cores in series can further cancel the remaining response. Two toroidal cores are switched between flux saturation levels, first one polarity then the other. If the cores exhibit matched and symmetric B-H characteristics, sense windings of the common polarity around each core produce equal and opposite signals. The output of either winding is non-zero only during that time in which the flux in the cores is changing (i.e., not saturated.) The sum of the two sense winding signals is zero (figure 1).

\subsubsection{Excitation with dc bias}

The beam in the pipe is considered a "single-turn" winding from the perspective of the DCCT system. The dc component of the beam biases each core with flux of the same polarity, causing an asymmetry in both cores' hysteresis curves. Since the flux in one core due to the modulator drive remains out of phase with that in the second, the flux in each core reaches saturation $180^{\circ}$ out of phase. The net result is a flux imbalance between the two cores, producing an output signal at even harmonics of the excitation frequency (figure 2 and figure 3). The magnitude of the second harmonic is directly proportional to the dc beam current. 




Figure 1. A Labview simulation models the magneto-modulator signals and hysteresis responses during pure, ideal AC excitation. With an ideal AC drive signal with no DC excitation, this scenario provides a "net-zero" case. Here, the induced signal on either core is solely due to the ideal sinusoid drive signal. However, with the cores excited in opposite phase, the frequency spectrum of the sum of cores'contributions, "Total Induced Signal V(t)," is zero. 


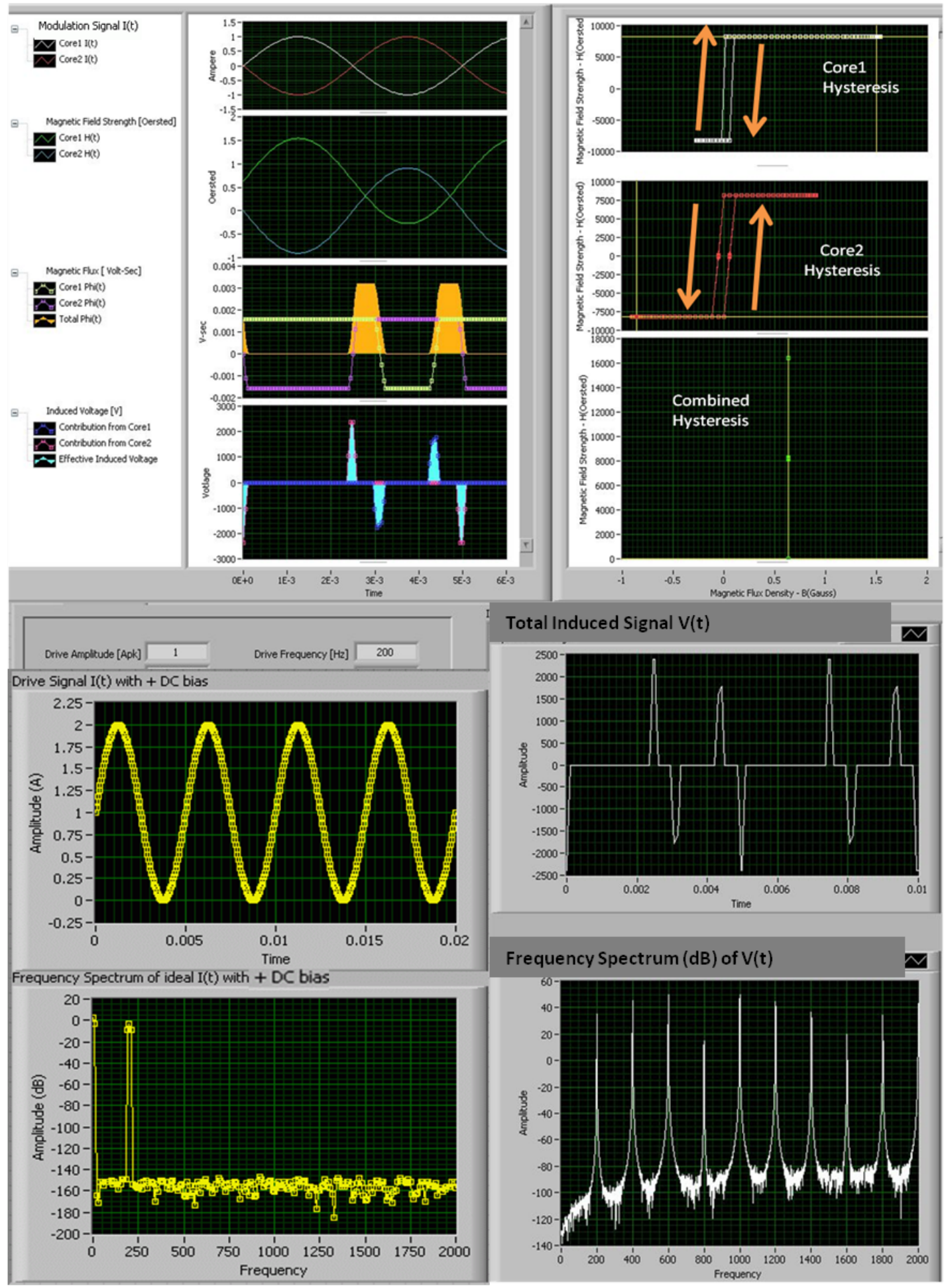

Figure 2. A Labview simulation models the magneto-modulator signals and hysteresis responses during pure, ideal AC excitation with a positive DC bias. Here, the sum of cores'contributions produced a positive flux density for all magnetic fields. The "Total Induced Signal V(t)" now contains even harmonics of the drive signal. 


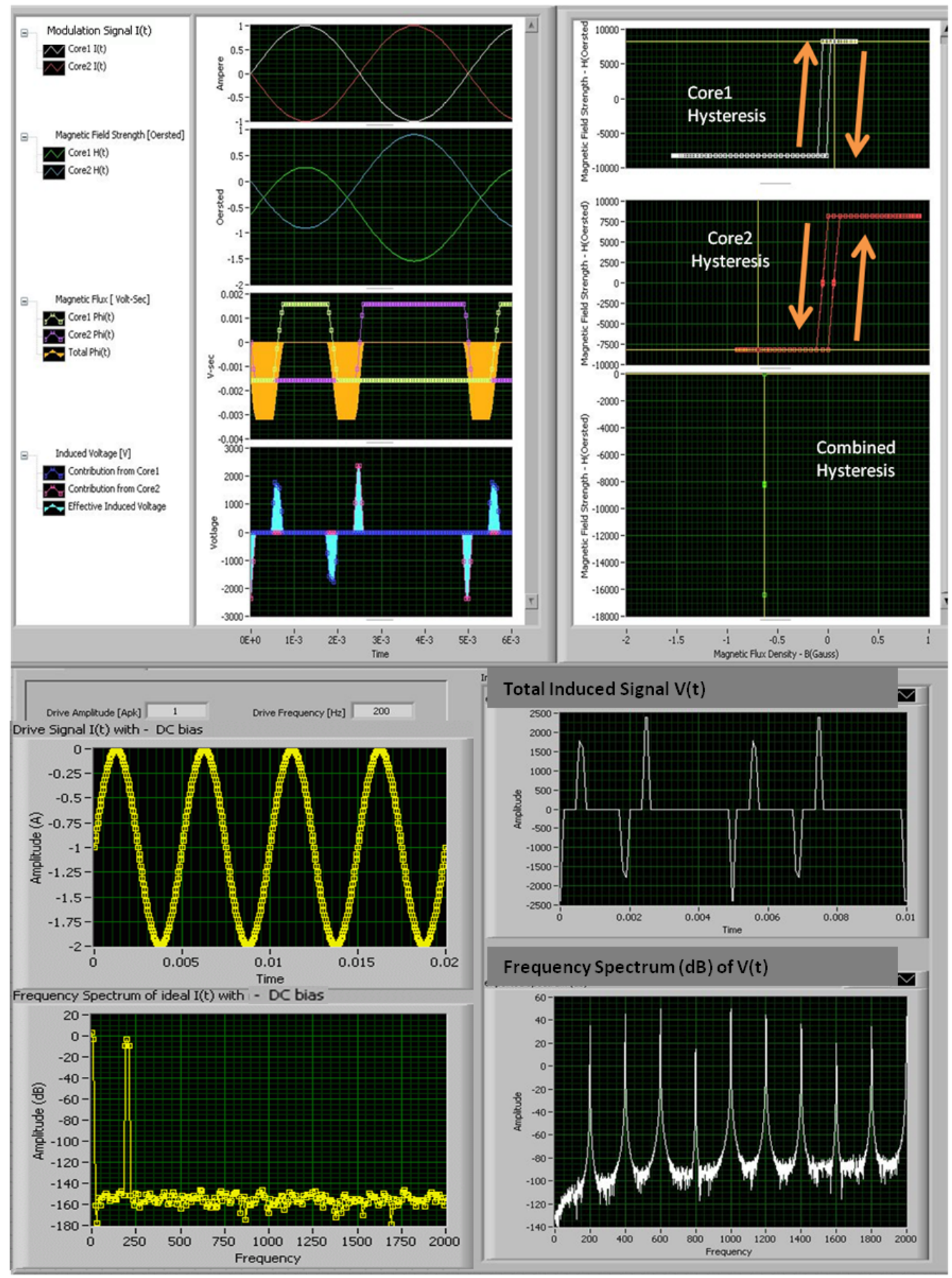

Figure 3. A Labview simulation models the magneto-modulator signals and hysteresis responses during pure, ideal AC excitation with a negative DC bias. Here, the sum of cores'contributions produced a negative flux density for all magnetic fields. The "Total Induced Signal V(t)" now contains even harmonics of the drive signal. 


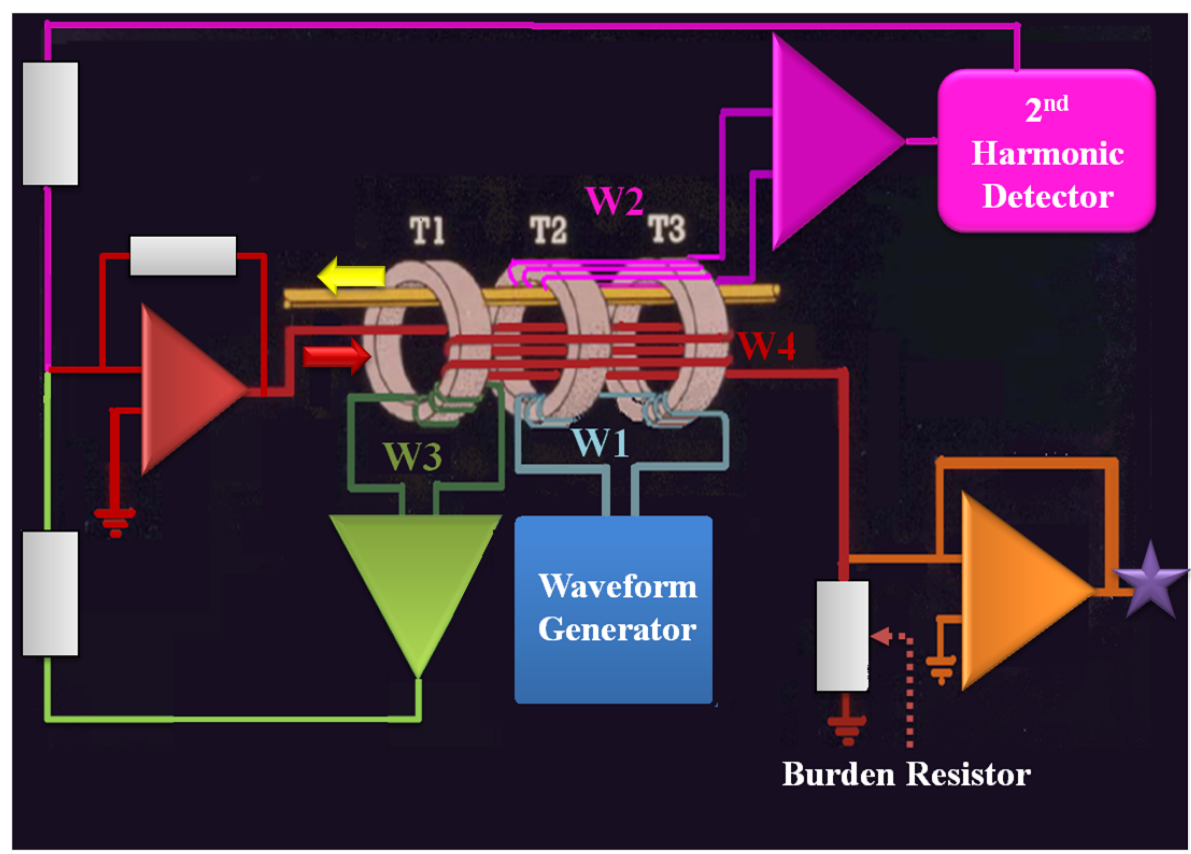

Figure 4. This figure depicts a simplified block diagram of the feedback loop to maintain net-zero flux. Cores T2\&T3 are modulated via drive windings (W1) at some excitation frequency. The magnitude of the $2^{\text {nd }}$ harmonic content of the induced signal at DC Sense windings (W2) is summed with the induced signal at the AC Sense windings (W3). The combined response generates a compensating current (red arrow), which is feedback through another set of windings (W4), cancelling out the effects of the beam current (yellow arrow). The compensating current is measured across a burden resistor (purple star).

\subsection{Application of bucking current}

The result of the second harmonic detector is used to generate a proportional bucking current, which is feed back to the detector via single-turn winding. An equal but opposing flux is produced to counteract the original change of flux caused by the beam, This path forms a feedback loop, which continually reestablishes the net-zero flux in the detector (figure 4). As it is directly related, the compensating current can be considered to be a true measure of the beam current, primarily limited by the transfer function of the closed feedback loop.

\subsection{Extending system bandwidth}

Furthermore, a second set of magnetic cores used to extend the bandwidth of the system.

Not modulated, this section of the detector would directly follow classical transformer theory. In an ideal transformer, the induced voltage in the secondary winding $\left(\mathrm{V}_{s}\right)$ is in proportion to the primary voltage $\left(\mathrm{V}_{p}\right)$, and is given by the ratio of the number of turns in the secondary $\left(\mathrm{N}_{s}\right)$ to the number of turns in the primary $\left(\mathrm{N}_{p}\right)$. Acting as a single-turn primary winding, beam currents will induce bandpass-filtered signals on a secondary set of pickup winding. Both the low and high corner frequency would be dominated by the characteristics of the transformers of the ac section.

Adding this induced signal to the result of the second harmonic detector allows the bucking current to be compromised of both ac and dc components. This is analogous to the 2-way crossover typology used in audio filters, where the constituent parallel highpass and lowpass adjacent filter 


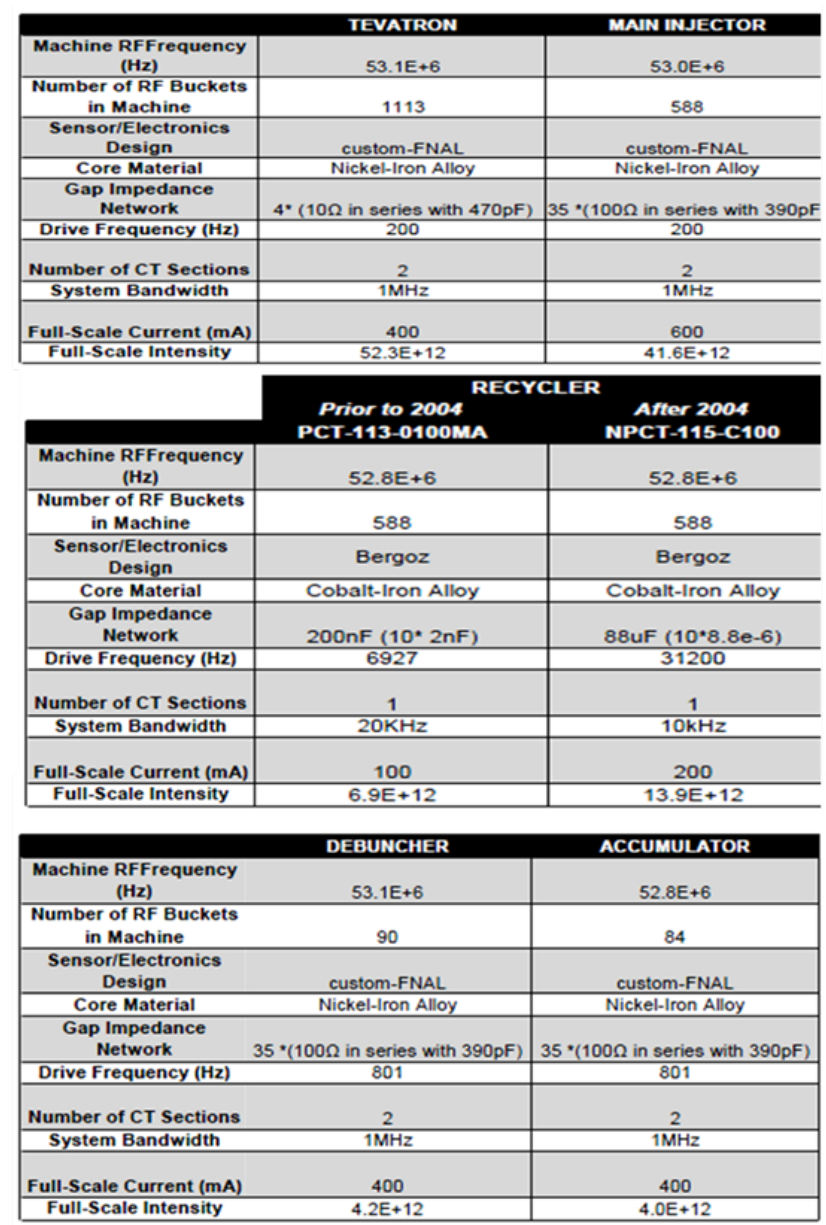

Figure 5. Summary of all DCCT installation at FNAL

sections yields a flat summed frequency response. Effectively, total frequency response would show that the bandwidth of the lowpass section would be extended to that of the highpass section.

This larger bandwidth allows the feedback current to cancel many of the rotation harmonics generated by the time structure of the beam. Without this bandwidth in the feedback, the modulated cores are balanced on average but can become heavily saturated one way or the other depending on the instantaneous beam current.

\section{DCCT systems installed at FNAL}

Although all DCCT systems operate under the same fundamental net-flux principle, the various installations have slight differences (figure 5). There are 2 main types of DCCT systems used at FNAL. One type was designed and built at FNAL (figure 6); it is installed in all the machines except the Recycler. In the Recycler, a commercial solution from Bergoz Instrumentation [10] was used (figure 7). However, in both systems, the interface into the FNAL control system is the same. 


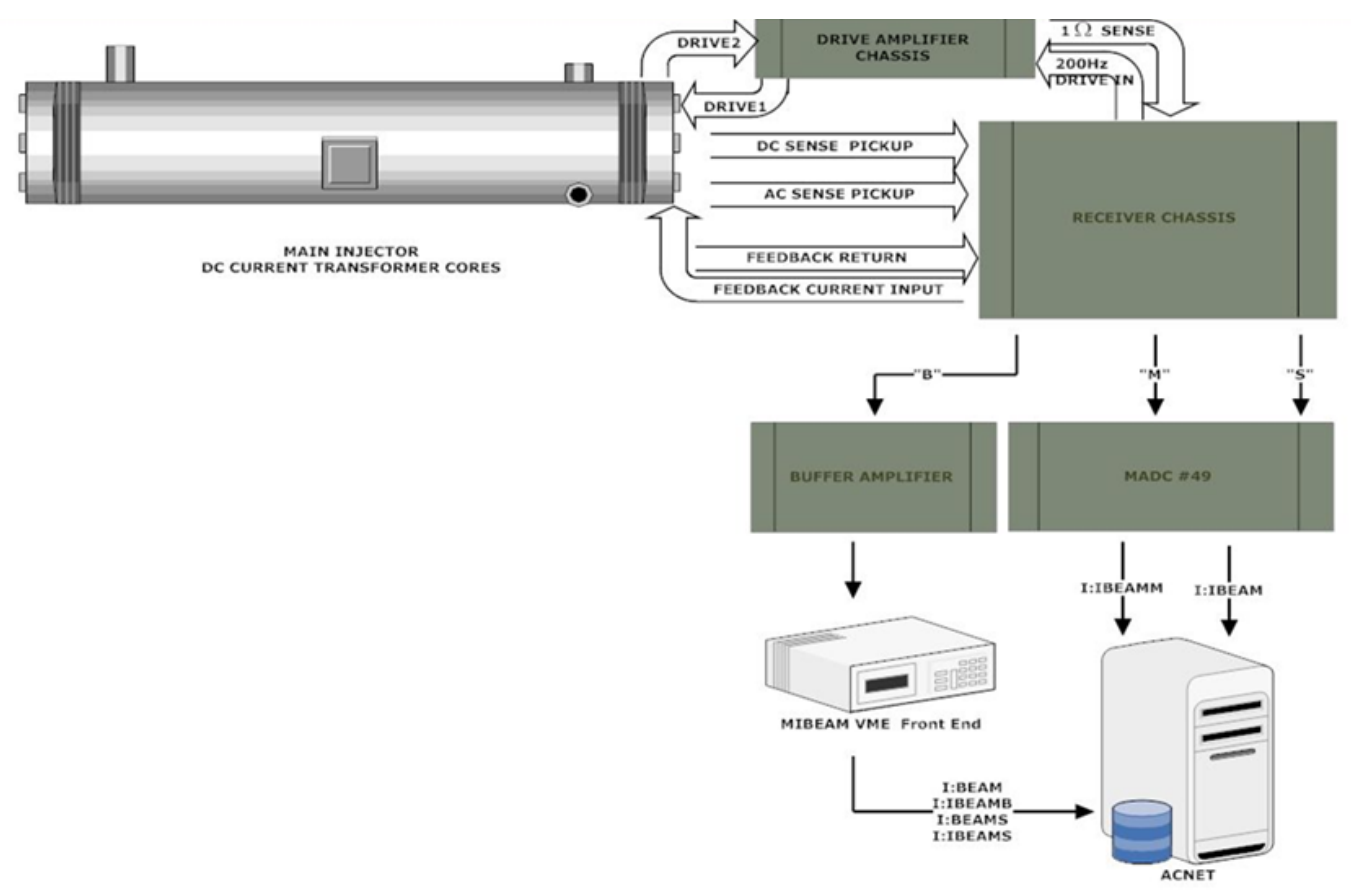

Figure 6. Block diagram of DCCT system, using custom-built sensor and electronics.
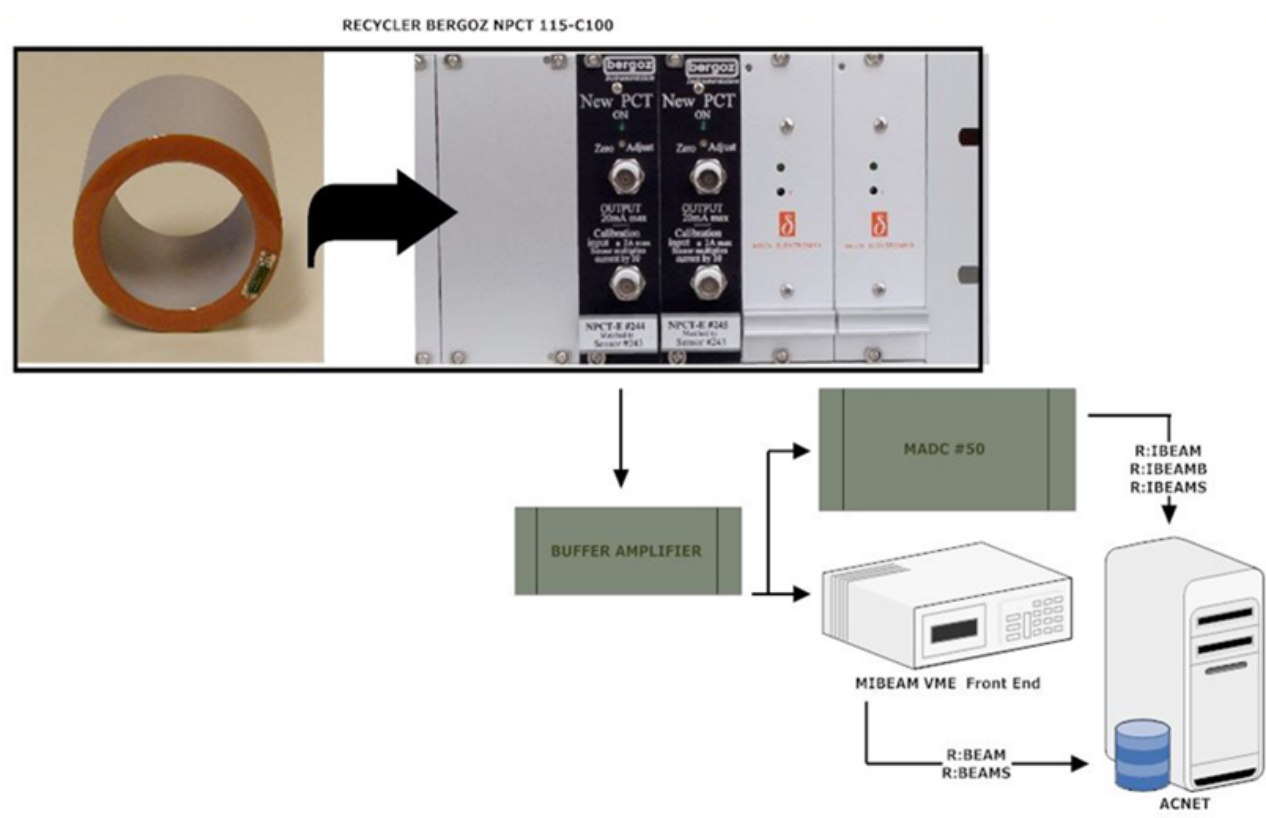

Figure 7. Block diagram of DCCT system, using a commercial sensor and electronics. 


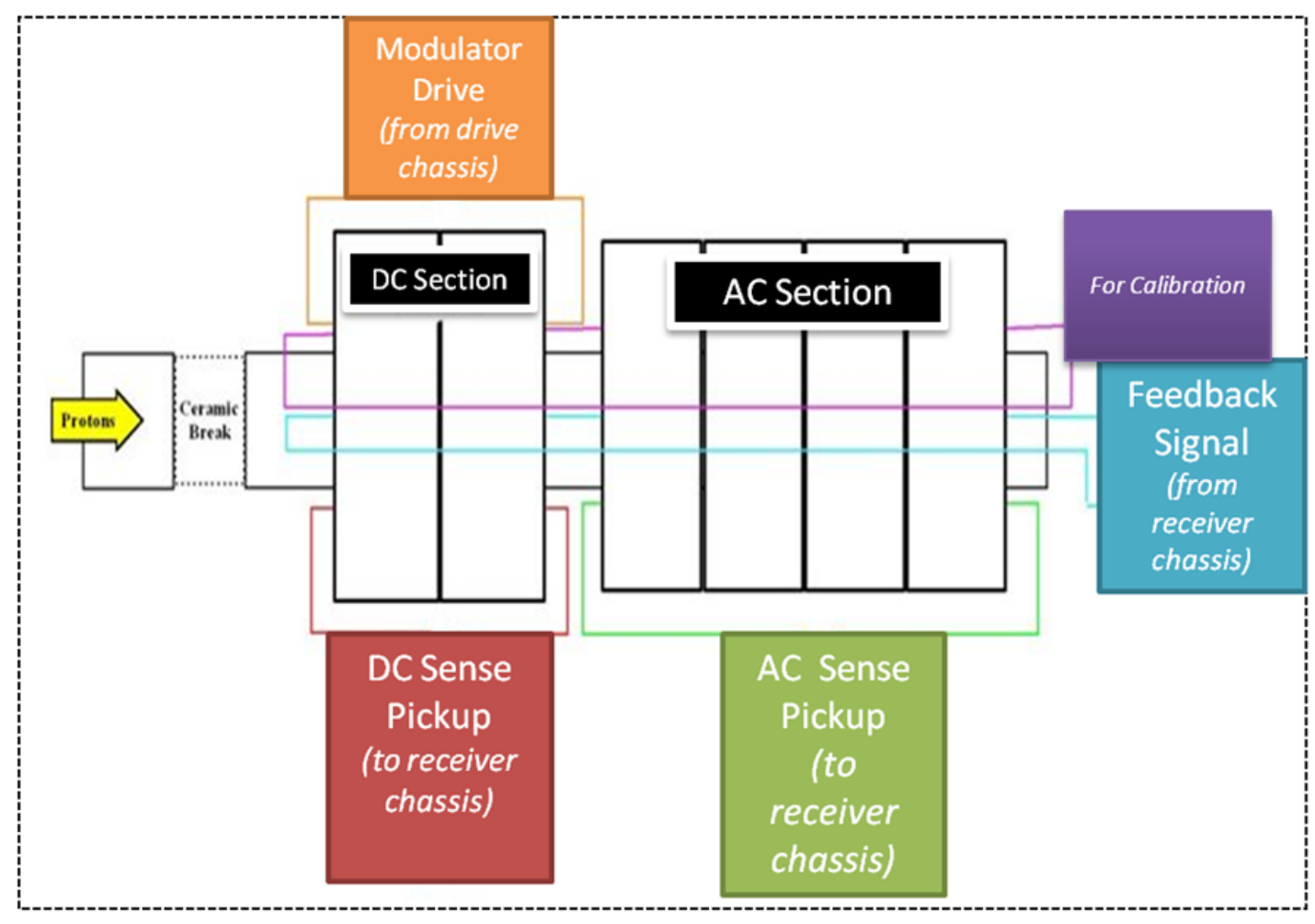

Figure 8. Exploded view of custom-built detector, previously shown in figure 6.

\subsection{Custom-built detector}

The detector housing is typically a steel shell that provides radiation shielding, noise immunity, and control of image currents. Inside, magnetic shielding is provided with three cylindrical metal layers. The layers are comprised of material, such as hipernorm, HYM-80, and 4.79 moly-permalloy, which all are highly permeable once annealed. The layers are separated with insulating 3/16"automotive felt. Multiple layers were used to increase the shielding factor. In addition, all components are mounted to keep the cores and connectors isolated from the beam pipe.

The custom-built detector contains 1 ceramic insulator and 2 sets of Magnetics [8, 9] Supermalloy tape-wound transformers (figure 8). Supermalloy is a nickel-iron alloy, with a minimum permeability of 45,000 .

Providing a break in the otherwise continuous beam pipe, the ceramic insulator's dimensions are based on the beam pipe diameter at each installation location. The core, the gap capacitance and the wall current bypass form together a cavity. A passive network is placed across the ceramic break to control the gap impedance as well as keep high harmonics out of the cavity. The passive network is installed by soldering pairs of leaded, precision passive components in parallel, between 2 steel cable ties. This RC "ladder" is then pressure- fitted across the gap using hose-clamps.

One set of cores, DC Section, consists of two toroidal current transformers (CT), with matched hysteresis curves. Each are oppositely wound with the same number of turns and connected in series. In the Main Injector and Tevatron, DCCT systems modulate their cores at $200 \mathrm{~Hz}$, using a HP6824A power amplifier. As the HP6824A became obsolete, it was replaced with an Apex 
PA07A power amplifier circuit. Although the circuit was functionally equivalent, design differences still lead to increasing the modulation frequency to $801 \mathrm{~Hz}$.

In addition, there is a secondary pickup winding around the DC Section, known as the DC Sense Pickup. Using the DC Sense Pickup, the second harmonic content of the total induced response of the CT pair can be detected. The magnitude of the resulting second harmonic measured effectively provides a feedback signal's dc component, which is proportional to the beam intensity.

The second set of cores, AC Section, is not modulated. Instead, there is a pickup winding around them, referred to as the AC Sense Pickup. The induced signal on these windings will provide the ac component to the feedback signal. The AC section also extends the system bandwidth to $1 \mathrm{MHz}$.

Processing of both the DC Sense and AC Sense Pickup signals take place in the receiver chassis electronics. After both open loop responses are added, the total resulting feedback signal is applied via a single-turn winding through all sets of cores, reestablishing net-zero flux in the detector.

The DCCT system determines the beam intensity by measuring the feedback signal across a high precision burden resistor. The full dynamic range of the system is proportional to the number of feedback windings and inversely proportional to the burden resistor's value.

A separate single-turn winding is used to provide a way to calibrate and test during no-beam situations.

\subsection{Commercial detector}

Originally, a Bergoz Parametric Current Transformer [7], PCT-113-0100MA, was purchased; this included the sensor, electronics, and cables. The sensor consists of a one set of amorphous nickelcolbalt alloy toroidal transformers, which are saturate at approximately 0.5 Tesla (5000 gauss). It operates under the same fundamental principles as DC Section in the custom-FNAL design, except with a much faster modulation frequency. Without the AC section, the PCT unit was limited to a $20 \mathrm{KHz}$ bandwidth.

A cooper housing was created to enclose the sensor and a ceramic insulator. The system worked satisfactorily until it was moved to in 2002. At the new location, the magnetic field from the now nearby Main Injector dipole magnet bus induced a $1 \times 10^{10}$ error in the measured beam intensity [8]. A magnetic cylindrical shield was constructed with 2 layers of 1/16" thick mu-metal, separated by an air gap (figure 9). Mu-metal is an alloy that presents a high permeability when it has been annealed. Closing the ends of a cylindrical shield with shielding disks, or flanges, adds very little to the shielding factor. The shield was 9" in diameter and 18 " long and reduced the magnetic field by $22 \mathrm{~dB}$ (figure 10).

In 2004, the PCT unit irrecoverably failed as the modulator core magnetic state became unstable. This instability occurs when one modulator core accumulates more remnant field than the other and the output drift occurs.

The failure may also have been aggravated by the higher beam currents and longer stores that prevailed toward the end of its life. The beam current contains very high peaks when the beam is bunched, as it is routinely in Recycler. The lack of ac section increases its inability to cancel higher frequency components in the beam. Furthermore, if the two modulator cores are not similar in permeability, the one with higher permeability will saturate earlier than the other. Consequently, the modulator sense winding, which collects the second harmonic of the modulator frequency, is 


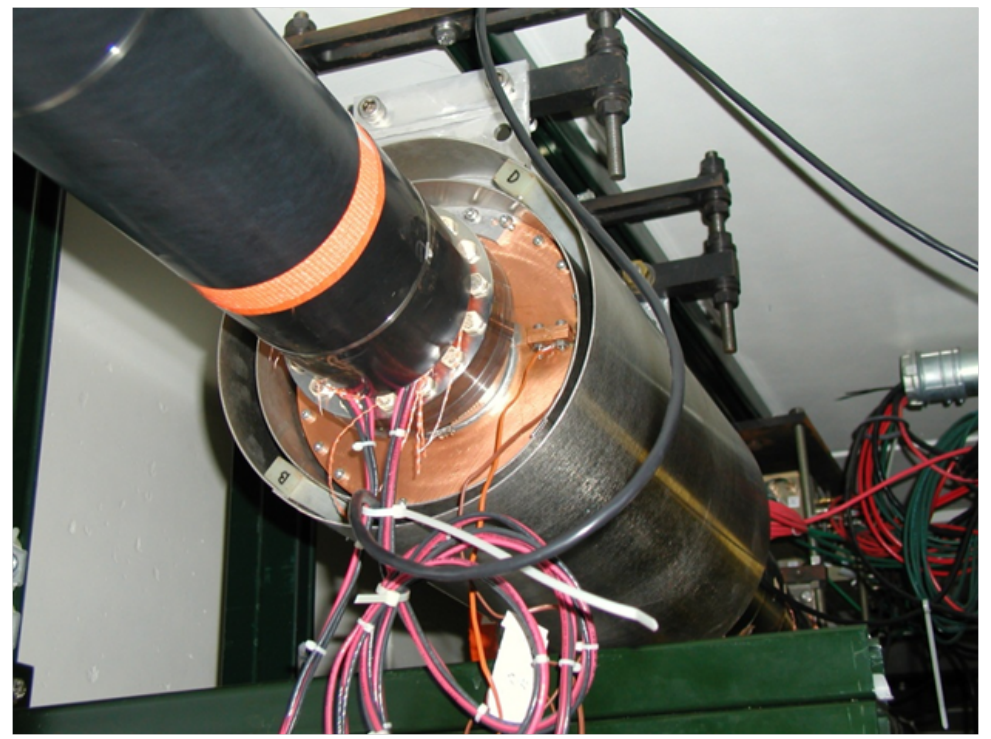

Figure 9. Picture of mu-metal shielding layers installed around the copper housing, containing the commercial detector. Multiple layers were used to increase the shielding factor.

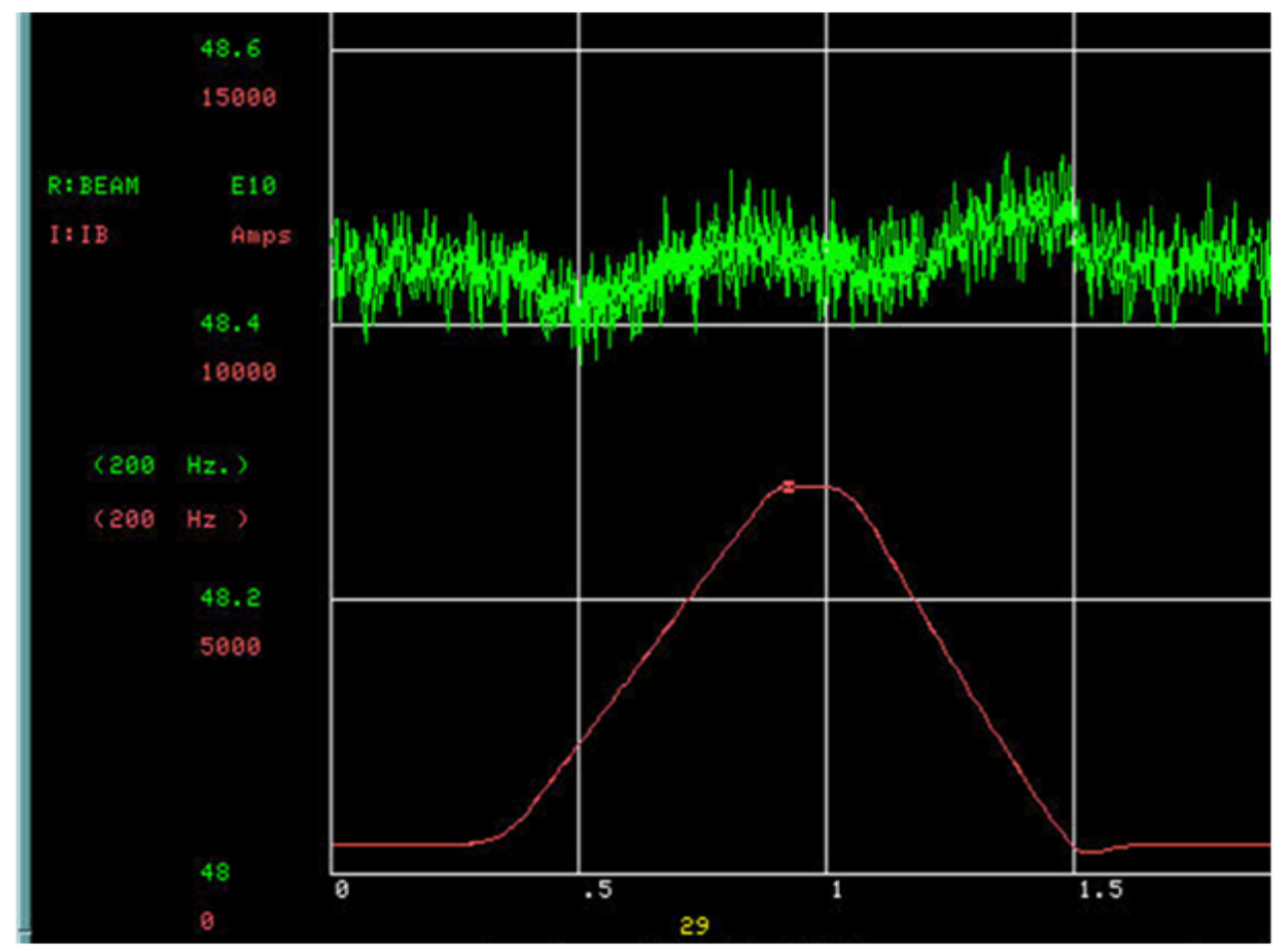

Figure 10. The plot depicts the final effect of the Main Injector dipole bus current (red) on the Recycler DCCT reading (green), with the mu metal shielding installed on the NPCT. The variation in the intensity is $\sim 0.05 \times 10^{10}$ particles compared to $1 \times 10^{10}$ before the shielding was installed. 
phase-shifted. Then, the synchronous demodulator is out of sync with the sense winding signal, and the demodulated signal is wrong. Essentially, the permeability of the two modulated cores became so different that the second harmonic generated with beam current could not be resolved.

Without any spare custom-detectors available, the Bergoz NPCT 115-C100 had to be purchased and installed as its replacement. Like the PCT model, the NPCT only has a DC section, and its system bandwidth is $10 \mathrm{KHz}$.

\section{Control system interface}

In all DCCT systems, the feedback signal is digitized, averaged, and scaled as an intensity measurement in a VME front end before interfacing with the Accelerator Controls Network (ACNET).

\subsection{VME environment}

A VME system node resides in close proximity to the receiver chassis outside the beam enclosure. It consists of a VME five-slot crate with a Motorola MVME-2401 controller, a 24-bit ICS-110BL8B ADC, and FNAL in-house support cards. The commercial ICS digitizer was measured to have 19.4 effective number of bits or $118.6 \mathrm{db}$ of dynamic range. The supporting cards consist of a Tevatron clock event (TCLK) decoder and a machine state (MDAT) decoder.

Virtually all accelerator timing depends in one way or another on the TCLK. TCLK can generate up to 256 fundamental events that are broadcast to all of the accelerators. Additional timing delays for triggering equipment can be derived locally through the use of application-specific decoder cards. The DCCT uses TCLK links to synchronize data acquisition of intensity measurements with the beam-related events in the machine.

MDAT is a link for communicating machine information across the accelerator complex in a real-time sense. The MDAT link currently supports 256 separate frames of machine data. Each of these frames is transmitted at a $720 \mathrm{~Hz}$ rate. The DCCT front-ends transmit scaled beam intensity as a 16 bit unsigned integer. The front-end can also read the MDAT frames transmitted by other front-end systems.

\subsection{Continuous intensity measurements}

The feedback signal is digitized by the ICS-110BL at $6.9 \mathrm{MHz}$. By using oversampling and decimation methods, the 24-bit sigma-delta A/D converter can achieve a dynamic range of $110 \mathrm{~dB}$ and 18.5 true bits. The digitized signal is available at a $720 \mathrm{~Hz}$ maximum data rate.

Since the feedback signal should be proportional to the beam intensity, this digitized output provides the intensity measurement after scale factors are applied. Such measurements are useful for beam transfer measurements and lifetime studies. In addition, it is used for all secondary calculations and covers the full range of beam intensities.

\subsection{Event-timed intensity measurements}

The VME front end can also record beam measurements on TCLK events. For these timedmeasurements, the front end provides two types of such read backs, fixed-event and variable-event.

For fixed-event read backs, the front end records 6 intensity measurements, coincident with predetermined sequential events after a machine reset. For example, if triggered on just a machine 
reset event, the following intensity measurements are generated: the intensity before the start of ramp; intensity at the start of ramp; the intensity at start of flattop; and 3 intensities taken during various transfer events which differ depending on the type of Main Injector cycle. This buffered acquisition allows the retrieval of the data by data loggers in a less time restrictive manner.

The second type is the variable-event read back. These read backs have a settable TCLK trigger and a settable delay (measured in seconds). With this type of read back, a single intensity measurement is recorded, synchronized with a machine event plus some delay.

\subsection{Beam loss-rate measurements}

At FNAL, the beam loss monitors (BLMs) are the diagnostics tool for detecting radiation rate localized near ion chamber installed near transport magnets. By monitoring radiation effects caused by any beam energy loss, BLMs provide useful beam-tuning information and help protect components from damage due to excessive radiation levels. However, these devices do not directly report amount of beam energy lost.

The DCCT front-ends provide this type of beam energy loss-rate measurement. This is calculated with a simple $\delta \mathrm{I} / \delta \mathrm{t}$, instantaneous rate of change in the report beam current from the DCCT at any given energy at which a particular machine is operating. After applying noise thresholds, this measurement is used in different ways.

In Main Injector, it is combined with the ring energy to get a measurement of beam energy loss (figure 11 and figure 12). This measurement presents an instantaneous view of instabilities caused by changes in the magnet currents. This is useful for beam-line turning, estimating the efficiency of the machine, and possible irradiation problems in the tunnel.

In the Tevatron, Recycler and Accumulator storage rings, this beam loss measurement is used as an instantaneous warning for instabilities that suddenly crop up in those machines. The frontends do this by generating TCLK events when they detect a sudden beam loss. These TCLK events are broadcast site wide and can be used for anything from generating beam-inhibiting alarms for operators to triggering data loggers to collect data.

\section{Operational performance}

The DCCT intensity measurements have provided reliable and stable data for a wide range of measurements. Although the requirements for each machine parameter were met during the initial commissioning phase, continual improvements have been made throughout Run II to maximize linearity and resolution while suppressing noise and sensitivity to longitudinal time structure.

\subsection{RMS noise observation}

The combination of several tasks throughout Run II targeted improving noise immunity. For the custom-detector, tasks varied from tuning of notch filtering section, aligning phase of pickup signals with demodulator clock signals (figure 13), tuning the crossover frequency in the feedback loop (figure 14), and adding common-mode filtering on the pickup cables, in custom-built system.

For all systems, another task was to redesign the system buffer amplifiers with better commonmode rejection, low noise figures, low offset drift, and low temperature sensitivity. 


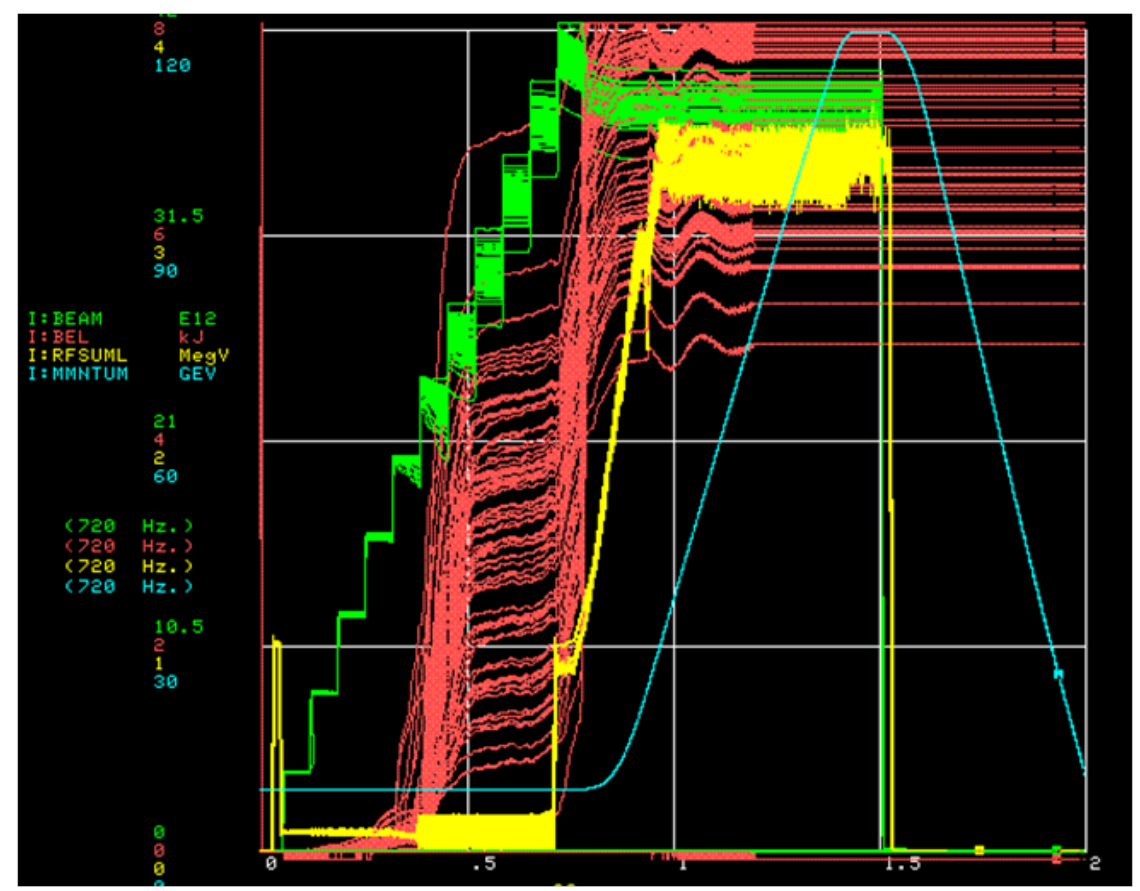

Figure 11. Time plot of the beam energy loss measurement (red trace-I:BEL) during Main Injector injection cycle. I:BEAM (green trace) represents the time plot of the beam intensity. Calculations for this cycle, in which $44.56 \times 10^{12}$ protons were injected, showed $4.11 \% 8 \mathrm{Gev}$ loss.

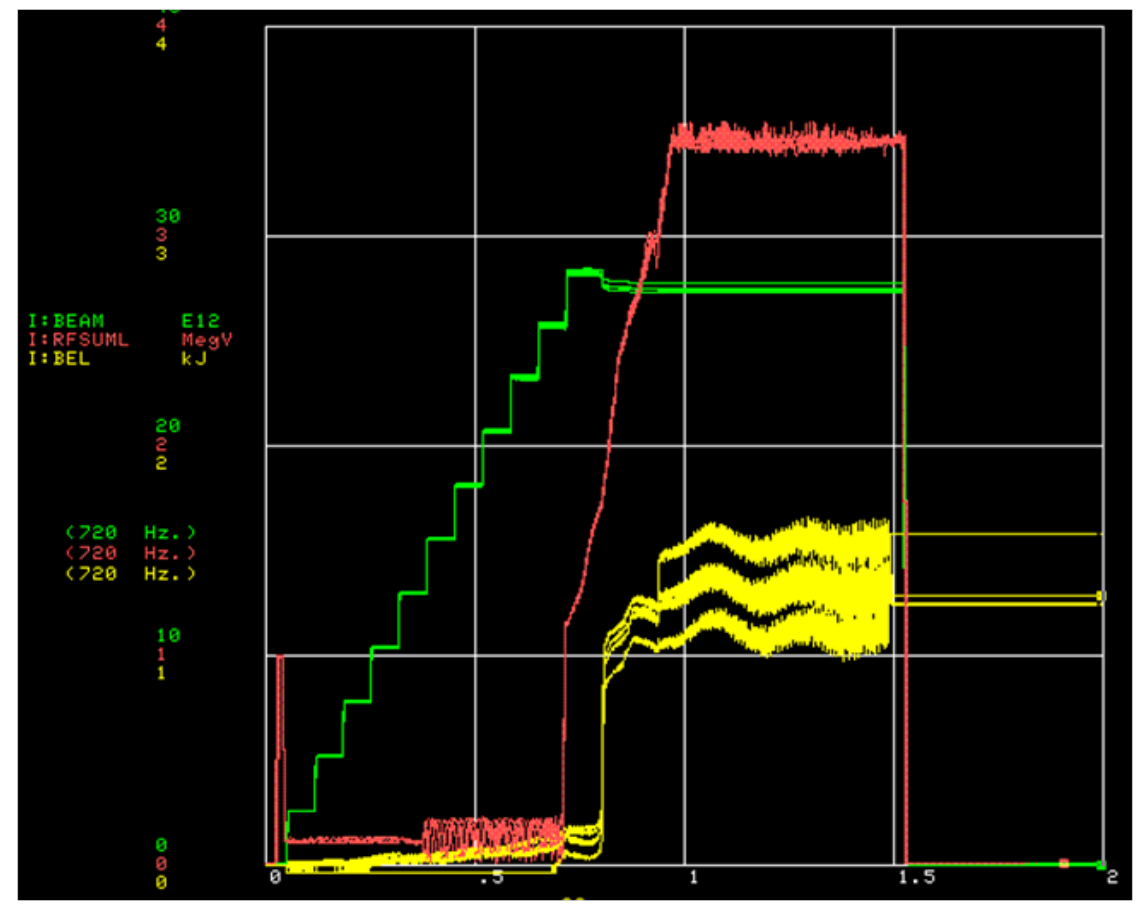

Figure 12. Time plot of the beam energy loss measurement (red trace-I:BEL) during Main Injector injection cycle. I:BEAM (green trace) represents the time plot of the beam intensity. Calculations for this cycle, in which $28.53 \times 10^{12}$ protons were injected, showed $0.40 \% 8 \mathrm{Gev}$ loss. 

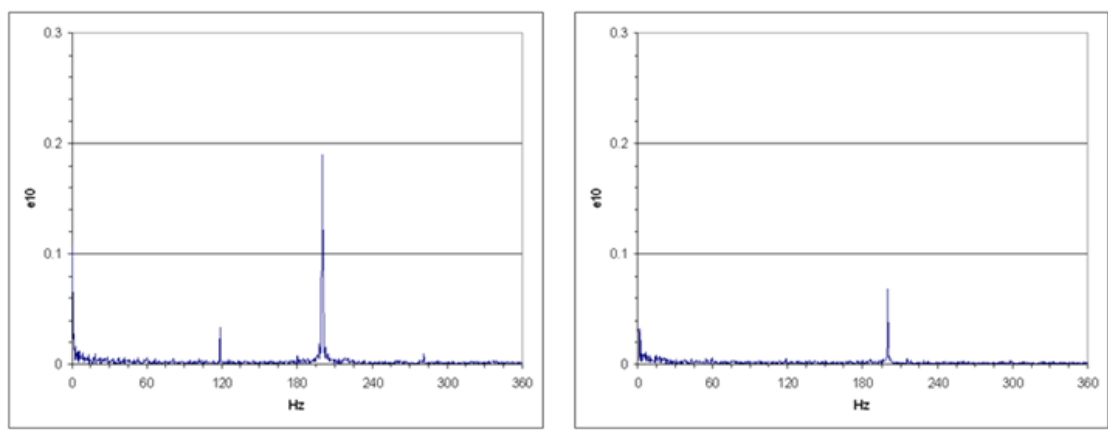

Figure 13. Measurement of frequency content of pickup winding before (left) and after (right) tuning notch filters and phase aligning in the Main Injector system. The $200 \mathrm{~Hz}$ noise component was reduced from $0.21 \times 10^{10}$ particles rms to $0.085 \times 10^{10}$ particles rms.

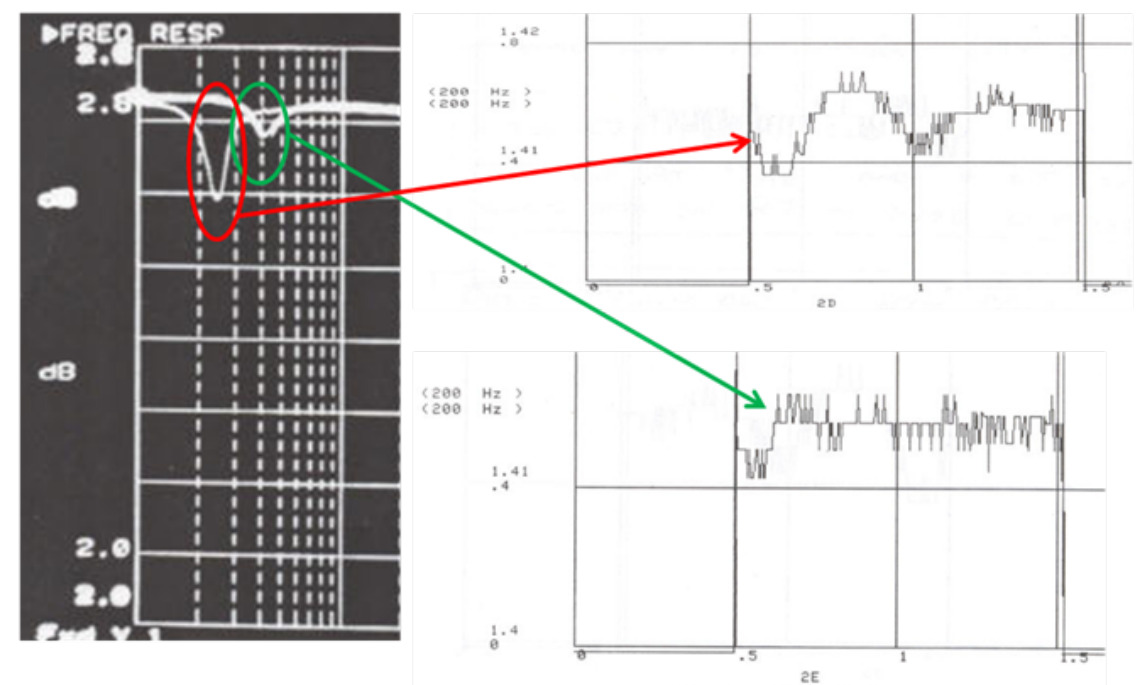

Figure 14. The sum of the AC section and the DC section in the closed feedback loop determine the depth and position of the crossover frequency. Adjusting the open loop response of each section minimizes the dip in the frequency domain, which translates to reducing the slow oscillation of the same frequency in the time domain. Such an adjustment was done in the Main Injector system, as shown in the difference between the red and green traces.

In addition, algorithms in the front end for all systems were continually revisited and improved. For example, a simple 12 sample boxcar average is implemented to further suppress power supply noise components at $60 \mathrm{~Hz}$ and its harmonics. Also, baseline-correction algorithms were implemented for accelerator, such as Main Injector and Debuncher, which receive periodic machine reset events.

A combination of these efforts resulted in the observed RMS noise levels listed in table 1.

\subsection{Nonlinearity studies}

DCCT calibrations involve simulating the beam current with a known current source. The intensity measurements are recorded and compared against expected intensities at given calibration 
Table 1. Observed RMS noise levels in the DCCT systems.

\begin{tabular}{|l|l|l|l|}
\hline Machine & Full-Scale Current & Full-Scale Intensity & Measured RMS Noise \\
\hline Recycler (NPCT) & $200 \mathrm{~mA}$ & $13.9 \times 10^{12}$ particles & $2.34 \times 10^{8}$ particles \\
\hline Main Injector & $600 \mathrm{~mA}$ & $41.6 \times 10^{12}$ particles & $1.36 \times 10^{7}$ particles \\
\hline Tevatron & $400 \mathrm{~mA}$ & $52.3 \times 10^{12}$ particles & $3.79 \times 10^{9}$ particles \\
\hline Debuncher & $400 \mathrm{~mA}$ & $4 \times 10^{12}$ particles & $1.08 \times 10^{7}$ particles \\
\hline Accumulator & $400 \mathrm{~mA}$ & $4 \times 10^{12}$ particles & $3.97 \times 10^{7}$ particles \\
\hline
\end{tabular}

Table 2. Nonlinearity errors in DCCT systems.

\begin{tabular}{|c|c|}
\hline Machine & \% Error At Full-Scale \\
\hline Recycler (NPCT) & $-0.04 \%$ \\
\hline Main Injector & $-0.39 \%$ \\
\hline Tevatron & $-0.65 \%$ \\
\hline Debuncher & $1.82 \%$ \\
\hline Accumulator & $-2.24 \%$ \\
\hline
\end{tabular}

currents. Because the feedback current is expected to be proportional to the beam intensities, it is expected that the regressions between the data sets should follow a linear model. A least-squares fit is performed between the data sets to find a simple "trend line", which minimizes the sum of the absolute values of the vertical "residuals" between points generated by the function and corresponding points in the data. The slope and offset of this trend line determine DCCT system scale factors, which are applied to the digitized feedback signal in the VME front end. All DCCT systems were calibrated at least once a year.

After a dedicated current source was purchased in 2005, a nonlinear distribution of the residual errors became more apparent. Unfortunately, the cause of this nonlinearity has not been determined. It is also unclear why the NPCT exhibit a much smaller error. Measurements using both a voltage source and a current source produce the same behavior, ruling out the calibration procedure itself as the cause. Consequently, calibration currents were limited to reduce errors at normal operating intensities of the machines. The maximum errors occur at full-scale intensities of the DCCT system (figure 15). Table 2 lists the nonlinearities found in all DCCT systems.

\subsection{Bunch length sensitivity}

The Recycler is a fixed energy storage machine that holds beam longitudinally in barrier buckets. Antiprotons are accumulated and added to the existing Recycler beam. As antiprotons are injected, the time structure of the beam changes. The NPCT from Bergoz Instrumentation has shown up to a $3 \%$ error that depends on the time structure of the beam.

The typical time structure of Recycler beam contains harmonics of the $89811 \mathrm{~Hz}$ rotation frequency. These high frequency components of the beam are suppressed with a capacitive network across the ceramic break The Bergoz manual advises that the gap capacitance should be more than $10 \mathrm{nF}$ and less than $220 \mathrm{nF}$. 

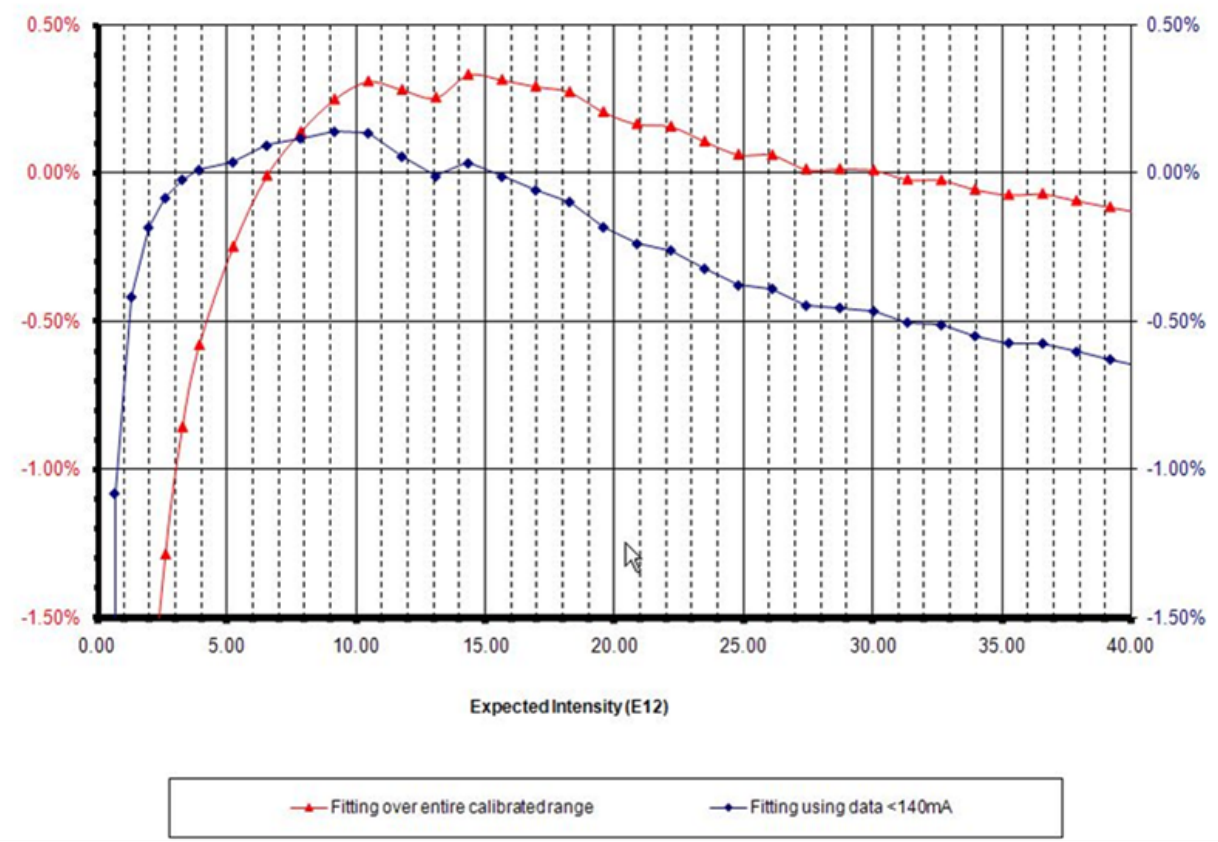

Figure 15. . The plot shows Tevatron DCCT errors as a function of intensity. The red trace reflects residual errors when linear model is based on a fit over the entire dynamic range. The blue trace reflects residual errors when linear model is based on a fit over just the lower half. Applying the fitting based on the blue trace, errors will be minimized for typical intensities of $1-2 \times 10^{13}$ particles seen in the Tevatron.

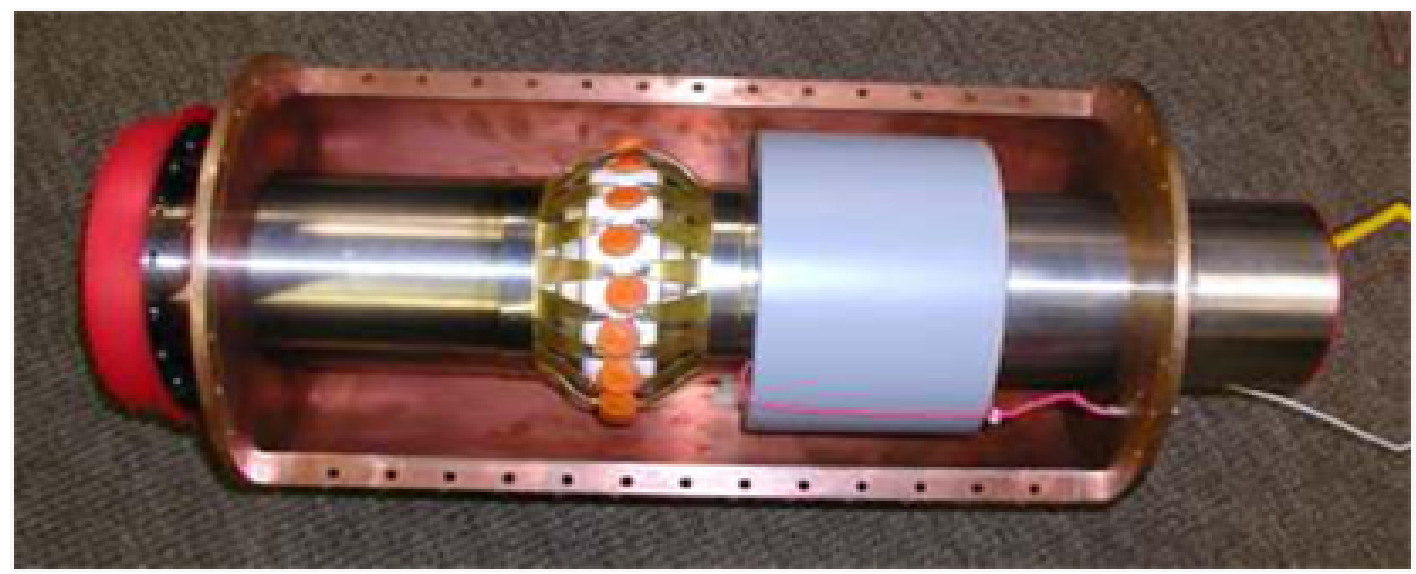

Figure 16. View of ceramic break, with final gap network of total 88uF, and Bergoz sensor inside Recycler detector.

The gap network was first modified to match the recommended maximum value. However, at the first rotation harmonic, $200 \mathrm{nF}$ is $8.9 \mathrm{ohms}$ and provides little or no effect. The capacitance has to be increased almost by a factor of 500 (figure 16) before actual beam measurements show substantial improvement (figure 17). An observable negative side effect has not been correlated to exceeding the recommended capacitance. 

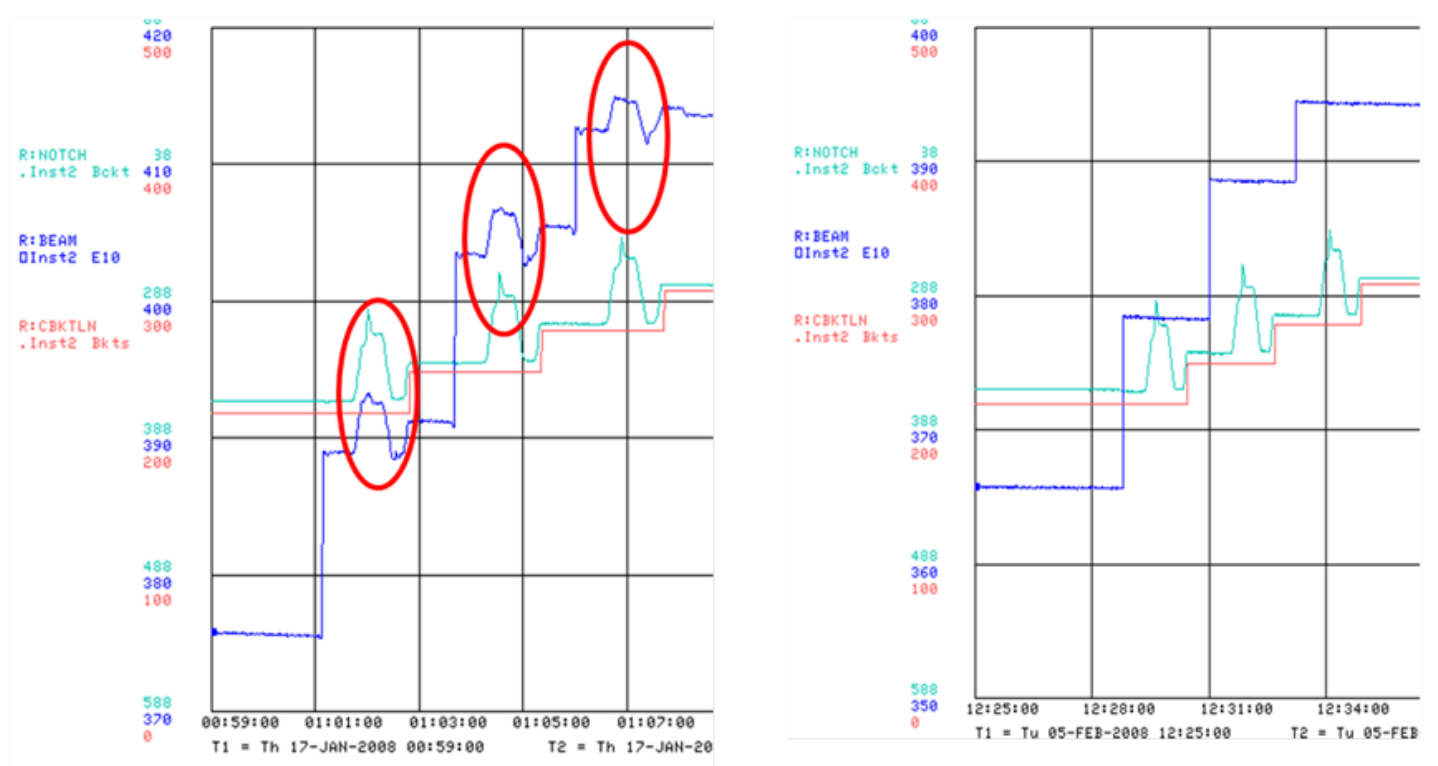

Figure 17. Plots of the Recycler DCCT intensity (blue trace) during 3 injections of $10 \times 10^{10}$ antiprotons. On the left plot, circled areas show errors of $5 \times 10^{10}$ with $200 \mathrm{nF}$ capacitance. The right plot shows vastly reduced errors with $88 \mu \mathrm{F}$ capacitance.

\section{Conclusion}

DCCT intensity monitors are important diagnostics tools used by operators to measure beam intensity in circulating rings. The devices were used for evaluating beam lifetimes, measuring beam transfer efficiencies, calculating beam energy losses, as well as broad spectrum instability monitors. During the Run II era, much effort was devoted to understanding the system's limitations and sensitivities as well as improving the system's resolution and reliability.

\section{Acknowledgments}

Contributions from the following are gratefully acknowledged: R. Webber, J. Crisp, B. Fellenz, G. Vogel, D. Heikkinen, T. Meyer and many others at Bergoz Instrumentation as well as FNAL Accelerator Division's Instrumentation Department, Controls Department, and Operations.

\section{References}

[1] R.C. Webber, Charged Particle Beam Current Monitoring Tutorial , in proceedings of $6^{\text {th }}$ Beam Instrumentation Workshop (BIW 1994), Vancouver, Canada, 2-6 October 1994.

[2] R.C. Webber, A Tutorial on beam current monitoring, in proceedings of $9^{\text {th }}$ Beam Instrumentation Workshop (BIW 2000), Cambridge, MA, 8-11 May 2000.

[3] R.C. Webber, A Tutorial on non-intercepting electromagnetic monitors for charged particle beams, in proceedings of $8^{\text {th }}$ International Meeting on Nuclear Applications and Utilization of Accelerators (AccAPP'07), Pocatello, ID, 30 July - 2 August 2007. 
[4] K.B. Unser, Toroidal AC and DC Current Transformers for Beam Intensity Measurements, Atomkernenerg/Kernt 47 (1985) 48.

[5] K.B. Unser, Design and Preliminary Tests of a Beam Intensity Monitor for LEP, in the proceedings of 1989 IEEE Part. Acc. Conf., IEEE Catalog No. 89CH2669-0.

[6] K. Unser, Beam Current Transformer with D.C. to 200 MHz Range, IEEE Trans. Nucl. Sci. 16 (1969) 934.

[7] K.B. Unser, The Parametric Current Transformer, A Beam Current Monitor Developed for LEP, in proceedings of $3^{\text {rd }}$ Accelerator Instrumentation Workshop, CEBAF, Newport News, VA, 28-31 Oct 1991, [AIP Conf. Proc. 252 (1992) 266, ISBN O-88318-934-8] [CERN SL/91-42 (BI)].

[8] J. Crisp, FNAL instrumentation: Lessons Learned, in proceedings of $10^{\text {th }}$ Beam Instrumentation Workshop (BIW 2002), Upton, New York, 6-9 May 2002.

[9] http://www.mag-inc.com/.

[10] http://www.bergoz.com/. 